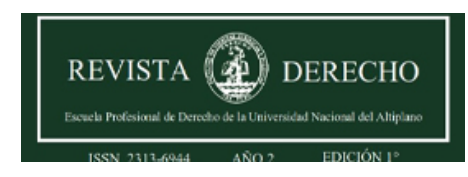

Revista de Derecho

ISSN: 2313-6944

ISSN: 2707-9651

revistaderecho@unap.edu.pe

Universidad Nacional del Altiplano

Perú

\title{
ETIOLOGÍA DEL DELITO DE TRATA DE PERSONAS EN SU MODALIDAD DE EXPLOTACIÓN SEXUAL Y LAS POLÍTICAS PÚBLICAS PARA SU PREVENCION EN LA REGIÓN DE PUNO
}

Flores Churqui, Heide Rosy; Mamani Arriola, Queila Tamar; Mamani Atamari, Erika Marycielo; Maquera Quispe, Deisy Yeshenia; Mamani Soncco, Hirayda Mariela; Vasquez Mamani, Cyntia Carolina ETIOLOGÍA DEL DELITO DE TRATA DE PERSONAS EN SU MODALIDAD DE EXPLOTACIÓN SEXUAL Y LAS POLÍTICAS PÚBLICAS PARA SU PREVENCION EN LA REGIÓN DE PUNO

Revista de Derecho, vol. 7, núm. 1, 2022

Universidad Nacional del Altiplano, Perú

Disponible en: https://www.redalyc.org/articulo.oa? id=671870939008 DOI: https://doi.org/10.47712/rd.2022.v7i1.172

\section{(c) (1)}

Esta obra está bajo una Licencia Creative Commons Atribución 4.0 Internacional. 


\section{ETIOLOGÍA DEL DELITO DE TRATA DE PERSONAS EN SU}

\section{MODALIDAD DE EXPLOTACIÓN SEXUAL Y LAS POLÍTICAS PÚBLICAS PARA SU PREVENCION EN LA REGIÓN DE PUNO}

\section{ETIOLOGY OF THE CRIME OF TRAFFICKING IN PERSONS IN ITS MODALITY OF SEXUAL EXPLOITATION AND PUBLIC POLICIES FOR ITS PREVENTION IN THE PUNO REGION}

Heide Rosy Flores Churqui

Universidad Nacional del Altiplano, Perú

floreschurquiheiderosy@gmail.com

(iD https://orcid.org/0000-0002-1503-8621

Queila Tamar Mamani Arriola

Universidad Nacional del Altiplano, Perú

queilatamararriola@gmail.com

(iD https://orcid.org/0000-0002-0768-2580

Erika Marycielo Mamani Atamari

Universidad Nacional del Altiplano, Perú

Erikamarycielo7@gmail.com

(iD https://orcid.org/0000-0002-3186-8828

Deisy Yeshenia Maquera Quispe

Universidad Nacional del Altiplano, Perú

deisyyeshenia110@gmail.com

(D) https://orcid.org/0000-0002-9621-1739

Hirayda Mariela Mamani Soncco

Universidad Nacional del Altiplano, Perú

HiraydaMarielaMamaniSoncco@gmail.com

(iD https://orcid.org/0000-0001-7431-6698

Cyntia Carolina Vasquez Mamani

Universidad Nacional del Altiplano, Perú

cynticarolinav@gmail.com

(iD https://orcid.org/0000-0002-7760-3067
DOI: https://doi.org/10.47712/rd.2022.v7i1.172

Redalyc: https://www.redalyc.org/articulo.oa? $\mathrm{id}=671870939008$

Recepción: 19 Agosto 2021 Aprobación: 04 Enero 2022 Publicación: 05 Enero 2022

\section{Resumen:}

El presente artículo tuvo como objetivos inferir la etiología primordial del delito de trata de personas en su modalidad de explotación sexual y analizar la aplicación de las Políticas Públicas referidas a la materia en la región de Puno, para ello se empleó dos cuestionarios que corroboraron la problemática que rodea a dicho delito, asimismo esto permitió concluir con la formulación de los lineamientos de las políticas públicas preventivas, para combatir el crecimiento abrumador del presente delito en tratamiento bajo su modalidad de explotación sexual.

Palabras Clave: Etiología, Explotación sexual, Trata de personas, Políticas publicas. 


\begin{abstract}
:
The present article aimed to infer the primary etiology of the crime of trafficking in persons in its modality of sexual exploitation and analyze the application of Public Policies on the matter in the Puno region, for which two questionnaires were used that corroborated the problem that surrounds said crime, this also allows us to conclude with the formulation of the guidelines of preventive public policies, to combat the overwhelming growth of this crime under treatment under its modality of sexual exploitation.
\end{abstract}

KEYWORDS: Etiology, Sexual exploitation, Human trafficking, Public policies.

\title{
I. INTRODUCCIÓN
}

La trata de personas en su modalidad de explotación sexual es un delito que vulnera gravemente la dignidad y libertad de las personas que a través de redes criminales que explotan de la peor forma al ser humano. Resulta lamentable que aún sigamos teniendo cifras relevantes de víctimas de este delito cuando tenemos organismos y una sociedad que debe mostrar el total rechazo al tolerar todo tipo de tarta de personas siendo el pilar suficiente para emprender un trabajo de investigación teniendo como objetivo inferir en la etiología de la Trata de personas en su modalidad de explotación sexual en la Región de Puno y analizar críticamente la aplicación de las políticas públicas a través del Plan Nacional De Acción Contra La Trata De Personas 2017 -2021 y el Plan Regional Contra la Trata de Personas 2018 - 2021 aprobado mediante Ordenanza Regional No 03-2018-GR PUNO-CRP.

Es la problemática latente que nos llevó a emprender esta investigación teniendo como primer objetivo el inferir en la etiología del delito de trata de personas en su modalidad de explotación sexual y como segundo objetivo el analizar la aplicabilidad de las políticas públicas. Lo cual nos permite afirmar que nuestro organismo público encargado de poner en marcha los planes aprobados conforme a la legalidad aún no efectivizan las políticas para frenar este delito. Es así que tras el análisis de los resultados pasamos a establecer lineamientos que coadyuven a la formación de políticas públicas preventivas. La comunidad académica se traza como objetivo el brindar los lineamientos a base de propuesta para que nuestros organismos con poder de acción los tomen en cuenta en su mesas de diálogo con entidades creando redes de trabajo para revertir la realidad de las víctimas y familias que vienen sopesando con esta impunidad; rescatar a nuestra población de este crimen es de carácter urgente no podemos seguir exponiendo a mujeres, niños y niñas a ser captadas por estas redes criminales.

\section{MARCO TEÓRICO}

\subsection{Delito de trata de personas en su modalidad de explotación sexual en Perú}

La trata de personas es la atracción, transporte, conducción o recepción de personas, haciendo uso de la intimidación, el engaño, el fraude o ya sea haciendo uso de la fuerza u otras formas de coacción para así obtener el asentimiento de una persona que tenga autoridad sobre otra, con el fin de realizar una explotación sobre la victima de la trata de personas, por ejemplo: explotación sexual, u obligarles a realizar trabajos o servicios de manera forzosa, someterles a la esclavitud o las prácticas análogas a ella, el servicio de servidumbre o hasta el extremo de la extracción de órganos.(ONU, 2000) El delito de trata de personas se caracteriza de tres elementos, medios, conductas y finalidades, donde la relación entre el agresor y la victima aprovechando el primero de la mala realidad esta persona, para aprovecharse del mal estado en que se encuentra y someterla a una situación de explotación y abuso de diferentes modalidades". (Chs- alternativo , 2010)

La trata con fines de explotación sexual: La explotación sexual es la utilización del cuerpo de una persona como un objeto sexual a cambio de ingresos económicos u otra clase de pago como educación, alimentación 
entre otros. En el Perú se favorece esa situación con la formalidad de locales dedicados a la explotación sexual con la fachada de Night Clubs o lugares de diversión nocturna ocasionando así un incremento de explotación sexual de mujeres, niñas y adolescentes donde se ejerce la prostitución y la explotación sexual comercial infantil. [1] Por ejemplo esto ocurre con notoriedad en zonas mineras donde se ve prostíbulos y jovencitas menores de edad que son captadas de diferentes regiones del Perú principalmente de orígenes andinos o quechua hablantes las mismas que son explotadas sexualmente obligadas, tan bien a trabajo forzado en bares, restaurantes esto en lugares donde hay una gran cantidad de hombres trabajando de manera legal o ilegal. (Chs- alternativo, 2010, pág. 13)

\subsection{Etiología del delito de trata de personas en su modalidad de explotación sexual}

\subsubsection{Economía}

Las causas económicas constituyen el conglomerado de los factores estructurales en materia de los delitos de trata de personas, en su modalidad de explotación sexual, ello debido a que los altos niveles de pobreza afectan directa y diferencialmente a mujeres, niños y adolescentes. Asimismo, la globalización, la cultura de consumo y las elevadas ganancias no solo promueven, sino también, facilitan y la compra, venta y comercialización respecto de los cuerpos de las personas, facilitando con ello la explotación sexual comercial.

\subsubsection{Sociedad de consumo}

Cuando se menciona las causas por las que incurren casos sobre trata de personas en su modalidad de explotación sexual, se puede dilucidar que una de las causas principales además de la económica, es la de la existencia de la sociedad de consumo, lo cual significa que existe una alta cantidad de consumidores y por ello existen redes organizadas para captar personas y satisfacer a los consumidores. Sin embargo, entendamos bien lo que es la sociedad de consumo; Bernal nos menciona acerca de ello y nos indica que a pesar de que un país se encuentre en desarrollo, una sociedad siempre se dejará influir por una sociedad de consumo. (Bernal, Varón, Becerra, Chaib, \& Archilla, 2013)

También corrobora este argumento Valero, quien nos indica que en una sociedad; los explotadores gozan de una mayor cantidad de capital económica lo cual les da la facilidad para extender una sociedad de consumo, lo cual Valero califica como un impulso que se tiene para generar un incremento en la trata de personas por su modalidad de explotación sexual. La sociedad de consumo genera que todo cuerpo se convierta en mercancía. (Lourido, 2018)

Cuando el autor Bernal menciona que una sociedad se deja influir por una sociedad de consumo, esto puede indicarnos que como consecuencia de la intensidad en el incremento de una sociedad de consumo es que existen las redes organizadas para esta captación, como corrobora la OIT, la cual nos indica que, en el proceso de captación, se encuentran diversos factores y uno de ellos es la existencia de redes organizadas para la captación de estas víctimas en la explotación sexual. (Bente, 2003)

\section{A. Propuestas laborales con falsas promesas}

Este puede ser el medio más recurrente para captar a las víctimas, como bien en una guía de trabajo nos mencionan que en estos últimos años los empleados encuentran un trabajo por contactos personales y que mientras mayor sea tu intención de buscar un empleo, más posibilidades laborales aparecen. (Yerro, 2017, pág. 13)

Lo importante de este medio es que por el hecho de que muchas personas buscan empleo, algunos lo hacen fuera de las agencias de trabajo, solo buscan redes sociales sin averiguar un poco más acerca del trabajo. Incluso algunas víctimas aceptan propuestas laborales fuera de su lugar de residencia, hablamos de ir en búsqueda de propuestas laborales en otros departamentos del país, los victimarios proponen salarios altos e incluso proponen empleos de horas muy cortas para que la víctima se vea interesada y así poder interceptarla.

\section{B. Seducción}


Este puede ser un medio por el cual el agente "victimario" consigue influir en la víctima, el autor Querol menciona que la seducción es un medio que se puede emplear por medio de cortejos, enamorar e incluso establecer vínculos sentimentales con la víctima, para poder manipularla y así conseguir un propósito y facilitar su captación o reclutamiento. (pág. 62)

Por ejemplo, en este caso sería conseguir la confianza de la víctima y así proponer a la víctima una serie de propuestas laborales, emprendimiento fuera de la ciudad donde ella reside, proponerle hechos que la víctima cree y así lograr llevarla con su consentimiento fuera del lugar donde ella reside para luego inducirla en una trata de personas en su modalidad de explotación sexual.

Algunas víctimas llegan a confiar tanto en su victimario que pueden llegar a creer todas las propuestas irreales que este hace con el fin de reclutarlas y captarlas.

\section{Amenazas}

Este medio se utiliza para poner presión a la víctima, para acorralarla y lograr intimidar fuertemente a la víctima.

Muchas veces una amenaza se logra con la intimidación a la víctima, como Querol mencionó, este acto de intimidación puede ser anunciando a la víctima un mal realmente grave contra la esta o incluso contra su familia. Este medio se utiliza para condicionar a la víctima de cometer hechos contra su voluntad, esta condición puede ser amenazando a esta con dañar e incluso asesinar a sus familiares si la víctima no hace lo que el victimario desea. (pág. 52)

\section{Secuestro}

Este medio es uno de los delitos que más afectan en una sociedad, como nos indica la autora a quien, parafraseándola, nos menciona que este delito se consuma en el preciso momento que se detiene vulnerando la voluntad a la víctima ilegalmente con la finalidad de incurrir a la víctima a una organización ilegal. (De Gonzáles, 2002, pág. 60)

Esta conducta que priva de la libertad a una persona, es el medio ilegal usado por los victimarios para privar a la víctima y hacer de ella una víctima participe de la explotación sexual.

\subsection{Normativas}

\subsubsection{Internacional}

A. Protocolo para prevenir, reprimir y sancionar la trata de personas, especialmente mujeres y niños, que complementa la Convención de las Naciones Unidas contra la Delincuencia Organizada Transnacional aquí nos señalan medidas que se deben adoptar para solucionar y erradicar este problema. (ONU, 2000)

Se nos especifica diversos puntos que los estados adheridos a este, deben contemplar en su normativa nacional, algo que se debe destacar es el Art 9, donde destaca la Prevención de la trata de personas en todas sus modalidades, explica que cada estado debe señalar las políticas, medidas y programas para solucionar dicho problema, esta debe ser recurriendo en específico a la cooperación bilateral o multilateral a fin de erradicar aspectos que se relacionen y creen este problema. Otro punto destacable son los parámetros legislativos que se proponen para forma una cultura preventiva y vigiladora.

B. Declaración de Nueva York para los refugiados y los migrantes: Los Estados adheridos tienen como misión y objetivo luchar contra la trata de personas y el tráfico de migrantes con su visión de erradicación, incluso señalando medidas específicas para ubicar e identificar a las víctimas de la trata de personas o las personas en riesgo de trata, para así lograr, buscar formas de apoyo para las víctimas de la trata de personas, ya sea en sus diferentes modalidades.

\subsubsection{Nacional}

A. Ley $\mathrm{N}^{\circ} 28950$, es contra la trata de personas y tráfico ilícito de migrantes: Esta relacionad con entes que tienen como base proteger a las víctimas de trata de personas, las cuales están en una posición de vulnerabilidad. 
Esta ley tiene como base central la protección integral de la víctima de trata de personas, donde el estado orientara todo su poder para la protección y asistencia integral de la víctima esto incluye diversos aspectos como la repatriación, asistencia médica, psicológica, social y legal, etc. Esta ley establece beneficios hacia la colaboración significativa en el ámbito de la criminalidad organizada

B. Código Penal Peruano: El delito de trata de personas en su modalidad de explotación sexual, está ubicado en el artículo 153-b del código penal. Se puede considerar a la explotación sexual, como aquella forma que está más apegada a lo que conocemos como a la trata de personas. Ahora bien, la explotación sexual vendría a ser entonces una actividad ilegal, que mediante la cual una persona (podemos decir que en su mayoría de los casos son mujeres mayores y menores de edad) es sometida de una forma violenta a actividades relacionadas con connotaciones sexuales, esto, sin su voluntad y/o consentimiento, por la cual un tercero recibe una remuneración económica.

Entonces debemos entonces entender que la explotación sexual es el aprovechamiento de una persona con respecto de persona que vendría a ser la víctima, todo esto con la finalidad de tener ingresos considerables, esto porque la cantidad de consumidores que hay en el mundo, los mismos que desean tener relaciones sexuales en su mayoría con niñas, niños, adolescentes, mujeres y hasta a veces hombres, creándose así a raíz de eso mafias que son organizadas para reclutar personas de distintos lugares del país, o hasta a veces de países diferentes.

Hoy en día, este delito está vigente en todos los rincones del mundo favoreciendo de esta manera a la trata de personas, las principales víctimas son mujeres adolescentes, niñas y niños, mediante el cual redes de criminales secuestran, amenazan, a niños(as) y las explotan hasta que estos no resulten atractivos sexualmente. Este delito estaría relacionado con el delito de secuestro, ya que muchas veces, secuestran (mujeres, niños y niñas) con el fin de explotarlos sexualmente. De la misma manera en muchos de los casos, las víctimas de trata de personas en la modalidad de explotación sexual, son aquellas que hayan sido amenazadas, engañadas (esto aprovechándose que sus víctimas tienen algún deseo de lograr un cambio en sus vidas o salir de la pobreza extrema) o hasta a veces que hayan sido seducidas (podemos decir sentimentalmente).

\subsection{Políticas Publicas}

\subsubsection{Plan nacional contra la lucha de la trata de personas 2017- 2021}

El presente plan se constituye como un verdadero y adecuado instrumento normativo para combatir la acuciante problemática de la trata de personas, puesto que esta política es el eje principal y orientador de la política criminal que se debe llevar a cabo respecto de esta materia, así mismo, establece los criterios y lineamientos criminológicos para su tratamiento.

De este modo, dicho plan se enmarca en una serie de principios, fundamentos y enfoques de carácter sistémico y organizativo que se sustentan en la complejidad del tema que estamos abordando en el presente trabajo, así mismo, para lograr su adecuada implementación y práctica es menester realizar un trabajo conjunto y multisectorial que implique al gobierno central, así como, a los diferentes gobiernos locales y regionales, al igual que las instituciones y organizaciones referidos a la materia, cabe mencionar que el trabajo a realizarse implica también y de forma necesaria a la sociedad en su totalidad puesto que ellos son los principales actores para poder prevenir y combatir la trata de personas.

\section{METODOLOGÍA}

La investigación que se realiza sobre "La etiología del delito de trata de personas en su modalidad de explotación sexual y las políticas públicas para su prevención en la región de Puno” es de tipo descriptivo, según Dankhe, citado por Hernández, Fernández y Baptista, 2001 "Los estudios descriptivos buscan especificar las propiedades importantes de personas, grupos, comunidades o cualquier otro fenómeno que sea 
sometido a análisis" pág. 60, dicha investigación tiene por objetivos; Inferir la etiología primordiales del delito de trata de personas en su modalidad de explotación sexual en la región de Puno y Analizar la aplicación de la política publicas contra la trata de personas en la región de Puno, estas dos implicancias tuvo como punto de partida la utilización de instrumentos de investigación en este caso fueron los cuestionarios, que ayudaron como soporte y sustento de ambos objetivos. El cuestionario es una técnica de investigación de campo, sirve para obtener información de la población materia de estudio o de alguna forma se ven vinculados directa o indirectamente con la investigación. Este instrumento, tuvo dos funcionalidades; el primer cuestionario ayudo a inferir las causas del delito y el segundo cuestionario ayudo a analizar la aplicación que tienen las políticas públicas referidas al delito de trata de personas en su modalidad de explotación sexual desde la case de conocimiento de las entes gubernamentales, en base a los datos obtenidos se pudo iniciar con la formulación de lineamientos para políticas públicas preventivas, puesto que la perspectiva que se debe tener es buscar soluciones antes de que se configure el delito.

\section{RESULTADO}

\subsection{Etiología del delito de trata de personas en su modalidad de explotación sexual}

OBJETIVO $\mathrm{N}^{\circ}$ 1: Inferir la etiología primordial del delito de trata de personas en su modalidad de explotación sexual en la región de Puno

Nuestra población de estudio consta de un total de 270(100\%) encuestados, 122(45.2\%) oscilan entre los 13 a 16 años de edad; mientras que $94(34.8 \%)$ tienen entre 21 a más años de edad y $54(20 \%)$ presentan una edad entre los 17 a 20 años. Respecto al género de los encuestados se observa que un 147(54.4\%) corresponde al sexo femenino de nuestros encuestados mientras que un 123(45.6\%) corresponde al sexo masculino.

\section{A. Economia}

Como se sabe una de las principales causas de la trata de personas con fines de explotación sexual, es la falta económica o inestabilidad económica, puesto que muchas de las personas en su mayoría mujeres sufren de una escasa economía, ya que, muchas veces no suelen encontrar trabajos adecuados, orillándoles así en la búsqueda de trabajos inadecuados y muchas veces caen las manos equivocadas, todo por querer conseguir un trabajo que aparentemente era adecuado para ellas, y aquí viene un punto importante ya que, no solo son mujeres jóvenes quienes son propensas a ser víctimas, sino, hasta niños y adolescentes, puesto que, en nuestra realidad son adolescentes y hasta niños los que están en búsqueda de un trabajo siendo víctimas potenciales para caer en manos de tratantes de personas con fines de explotación sexual.

La falta de economía es una de las causas de la trata de personas con fines de explotación sexual, ya que en nuestra realidad es una gran mayoría de la población quienes son de escasos recursos, siendo entre ellos jóvenes, adolescentes, señores y se puede decir hasta niños, en el último caso son aquellos niños en situación de abandono o en peor de los casos es que son los propios progenitores quienes obligan a estos niños a buscar trabajo. Ahora bien, el problema inicia cuando al momento de la búsqueda de trabajo se dejan llevar en muchos de los casos por los beneficios o sueldo que dicho trabajo brinda, sin tener que investigar o indagar más sobre dicha propuesta laboral. Dicho lo anterior una de las modalidades por la cual las redes de tratantes de personas que captan a sus víctimas son mediante las propuestas laborales tal como lo señala la Comisión Europea en su manual para conocer el problema sobre trata de personas en el que indican que los tratantes publican avisos de trabajos y la información que consigna en ellas es parcial, puesto que esconden el verdadero fin de dicha propuesta laboral, muchas veces derivando así en explotación sexual. (Comision Europea, 2010) 


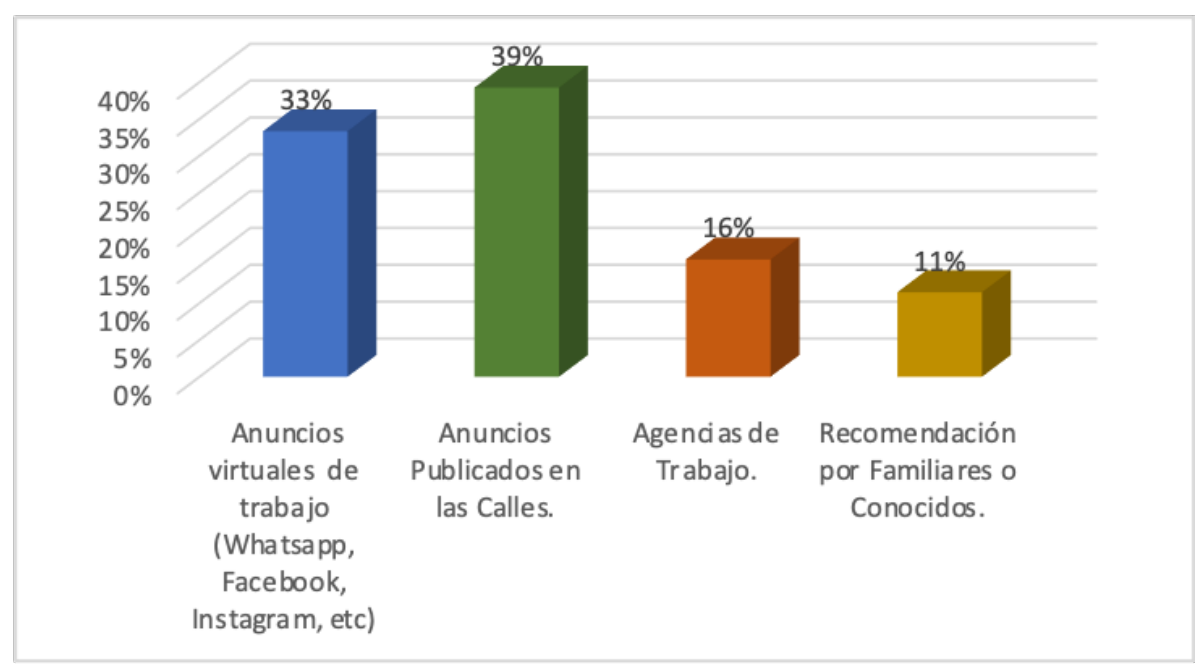

FIGURA 1

Generalmente, cuando usted esta en búsqueda de un trabajo ¿a donde recurre para solicitar dicho trabajo? Nota. Encuesta elaborada por el equipo de investigación y realizada mediante Google Forms, Julio 2021.

\section{Interpretación}

Según la figura 3, el 39,3\% de los encuestados, generalmente buscan trabajo mediante anuncios virtuales de trabajo, esto incluye lo que son las redes sociales, así como WhatsApp, Facebook, Instagram, entre otros; ya que, vivimos en la era de la tecnología y todo ahora es de manera virtual. Seguidamente el 33,3\%, de los encuestados respondieron que lo hacen mediante recomendación de familiares o conocidos, después el 15,9\% de los encuestados, emprenden la búsqueda de trabajo mediante agencias de trabajo y por último el 11,5\% de los encuestados lo hacen mediante anuncios publicados en las calles.

De lo anterior expuesto se puede inferir que una gran parte de la población cuando estos están en búsqueda de algún trabajo realizan la búsqueda mediante anuncios virtuales, lo cual no sería muy seguro a nuestro parecer, puesto que, una de las modalidades que justamente aplican aquellos que se dedican a la trata de personas, lo hacen captando mediante anuncios de trabajo de manera virtual, ya que esto conlleva al engaño hacia la víctima, es decir, la víctima es propensa de caer fácilmente en las manos de estos inescrupulosos. Seguidamente, también otra parte de la población los cuales realizan la búsqueda de trabajo mediante recomendación, ya sea de familiares o conocidos, esto por el hecho de que se sienten más seguros cuando el trabajo es recomendado por un familiar o conocido, ahora bien, es bueno saber que, aunque sea una pequeña parte de la población, si busquen trabajo en agencias, esto porque a nuestro parecer es más seguro, ya que, las agencias serian un medio más seguro de buscar trabajo, puesto que, ellas investigan previamente, tanto a empleadores como a los futuros empleados. Y por último una minoría de los encuestados buscan trabajo en anuncios publicados en las calles, esto a nuestra consideración, podría ser un poco preocupante, ya que no se sabe con certeza la finalidad de muchos de los trabajos, es decir, hay anuncios que solicitan personal, pero que no para la misma ciudad en que son publicados, sino, por el contrario, son en zonas alejadas a esta; y esto puede ser realmente preocupante.

\section{B. Sociedad de consumo}

Las redes criminales tienen mecanismos colaborativos para cometer el delito de trata de personas en la modalidad de explotación sexual, estas se forman con el objetivo de satisfacer la sociedad de consumo en referencia al acto sexual, es por tal motivo que para captar víctimas buscan formas o modos, los más comunes son; la seducción, amenaza o secuestro. Según el cuestionario aplicado a posibles víctimas se ha ratificado las formas como se llega a cometer el delito.

En la trata de personas en su modalidad de explotación sexual, la sociedad de consumo según el plan regional de acción contra la trata de personas de Loreto 2013-2017, hace alusión a un cliente sexual donde tan 
bien debe considerársele como el principal explotador sexual, pues si no hubiese un cliente sexual es decir no habría personas que pagan por obtener sexo con las víctimas de este delito, en consecuencia no habría razón para que estas redes criminales hagan un reclutamiento forzoso a más víctimas usando diferentes medios como el secuestro, ofreciendo falsas promesas de trabajo, por seducción, y amenaza constante entonces existen muchos factores que de alguna manera promueven la existencia y mantenimiento de este delito pero nos parece que esta sociedad o este elemento debido a su necesidad favorece a que las redes criminales quieran satisfacer la demanda del mercado (págs. 72-73).

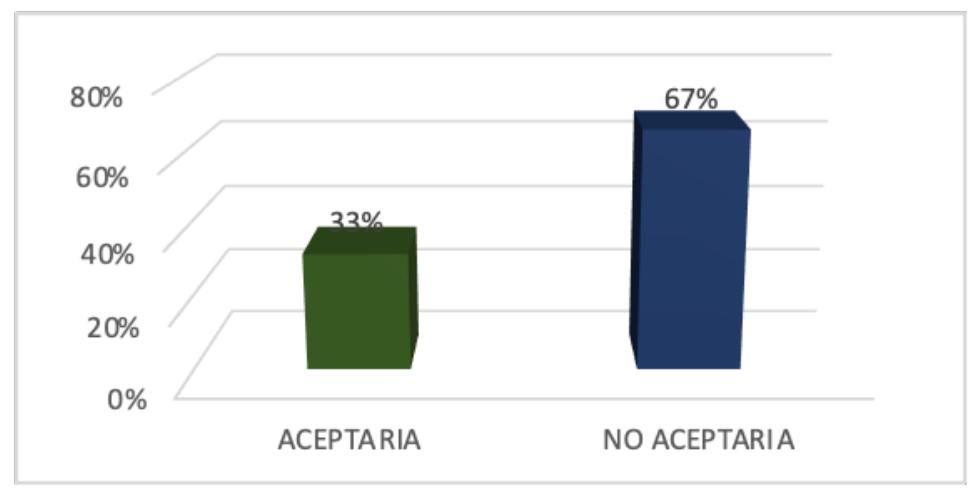

FIGURA 2

Caso Referencial

Tamar empieza una relación amorosa con Sebastián, a quien conoció en una reunión de estudios, este según Tamar tiene diversas cualidades; es agraciado, atento, de buena presencia y muy hablador. Sebastián le propone a Tamar realizar un viaje de vacaciones a Madre de Dios. Usted en la posición de Tamar: Nota. Encuesta elaborada por el equipo de investigación y realizada mediante Google Forms, Julio 2021.

\section{Interpretación}

Según la figura 2 , respecto al caso propuesto de los 270 encuestados tanto varones y mujeres señalaron que un $67 \%$ no aceptaría la propuesta de Sebastián y el 33\% aceptaría dicha propuesta de vacaciones , sin tener presente las circunstancias.

Las redes criminales para satisfacer la necesidad de la sociedad de consumo utilizan el modo o forma de seducción para cometer el delito. La seducción o enamoramiento es el medio por el cual el agente "victimario" consigue influir en la víctima, el autor Querol menciona al respecto que se puede emplear por medio de cortejos, enamorar e incluso establecer vínculos sentimentales con la víctima, para poder manipularla y así conseguir un propósito y facilitar su captación o reclutamiento. (pág. 62) Algunas víctimas llegan a confiar tanto en su victimario que pueden llegar a creer todas las propuestas irreales que este hace con el fin de reclutarlas y captarlas. En este caso se consiguió la confianza de la víctima, hasta proponerle unas vacaciones para luego inducirla en una trata de personas en su modalidad de explotación sexual según el informe de la Defensoría Del Pueblo efectivamente menciona que muchas veces los victimarios son sus parejas, en este caso los tratantes utilizaron la seducción y el enamoramiento para captarlas luego trasladarlas de su lugar de origen a otros lugares apartándolas de sus familiares. (págs. 44-46) 


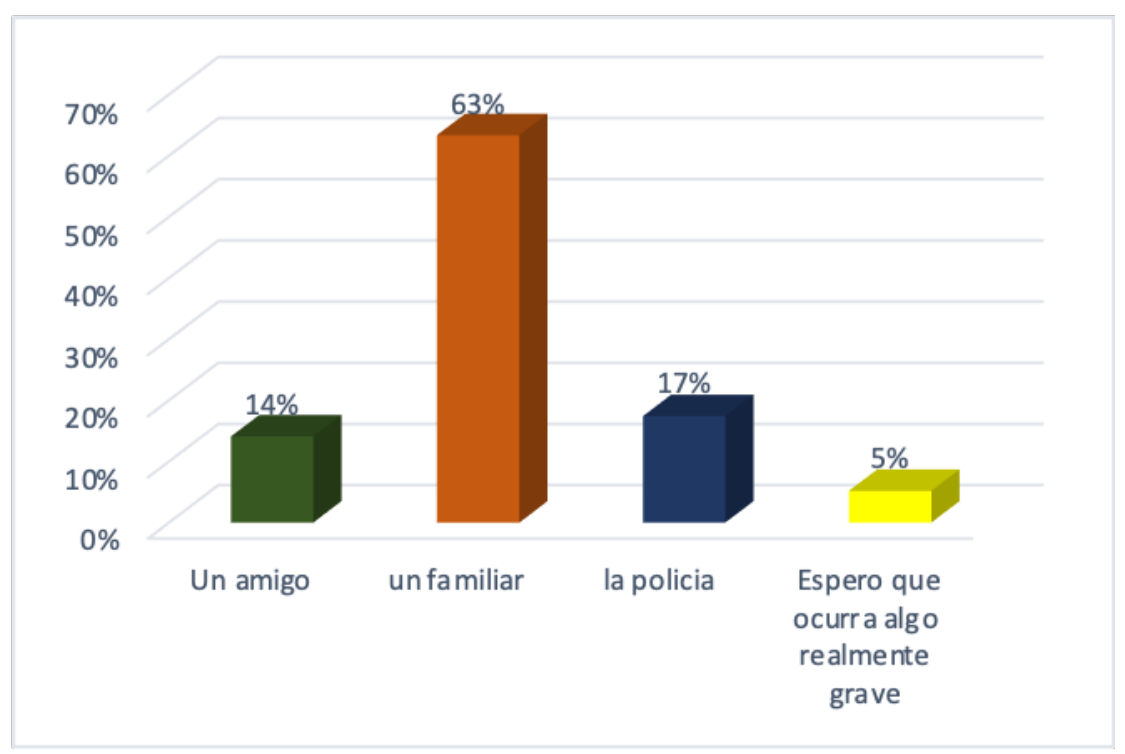

FIGURA 3

Ante una situación de amenaza, usted recurre primero a

Nota. Encuesta elaborada por el equipo de investigación y realizada mediante Google Forms, Julio 2021.

\section{Interpretación}

Ahora en base del informe de la defensoría del pueblo el sometimiento y el control de la víctima que realizan las redes criminales después de que esta ya ha pasado el siglo del traslado para luego encontrarse en el lugar destino a explotación sexual para poder satisfacer las necesidades de la sociedad de consumo en específico del cliente sexual abre puertas a la amenaza como un método de control donde el cual el victimario lo utiliza para lograr una intimidación hacia la víctima, haciendo uso de la violencia psicológica física y sexual palabras que atentan contra sus propias vidas, si estos intentan escapar o incluso contra las vidas de sus familiares. (págs. 46-48)

Querol mencionó, este acto de intimidación puede ser anunciando a la víctima un mal realmente grave contra esta o incluso contra su familia. Este medio se utiliza para condicionar a la víctima de cometer hechos contra su voluntad, esta condición puede ser amenazando a esta con dañar e incluso asesinar a sus familiares si la víctima no hace lo que el victimario desea. (pág. 52) Por ello es muy de difícil ya querer escapar de estas redes criminales que ponen a las víctimas en situaciones de amenaza constante y la pregunta es èsi se encuentra ante una situación de amenaza, donde recurriría específicamente? Cuestionario que se realizó en el cual según el grafico estadístico el $63 \%$ aseguro que recurriría a un familiar, un 17, \% a la policía, un $14 \%$ a un amigo y el $6 \%$ espera que ocurra algo realmente grave, entonces podemos asegurar una vez más que la familia es el lugar donde encontramos la confianza y seguridad, porque quizás la victima tiene ese temor a ser señalada y criticada por la sociedad. La policía tan bien cumple un rol fundamental al perseguir a estas redes criminales pero nosotros no practicamos la política de denunciar. Porque lo primero que hacemos es contárselo a alguien, un amigo o un familiar antes que al ente policial. 


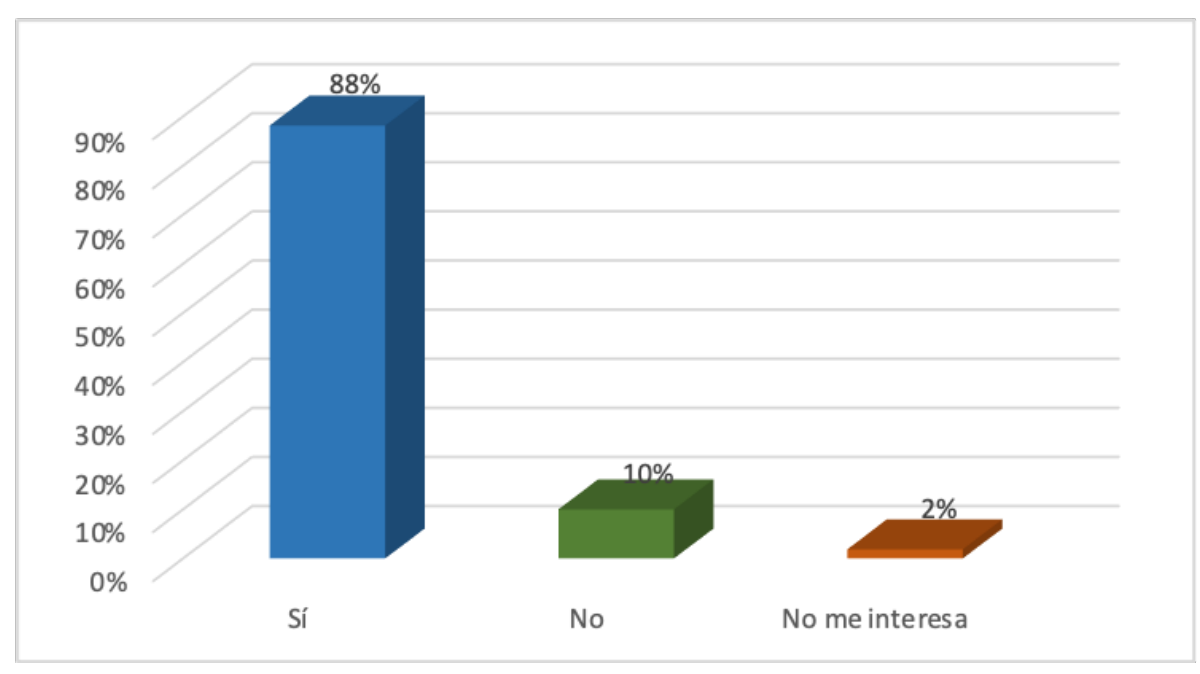

FIGURA 4

¿Conoce usted de qué trata el secuestro por trata de personas?

Nota. Encuesta elaborada por el equipo de investigación y realizada mediante Google Forms, Julio 2021.

\section{Interpretación}

Según la figura 4, el $88 \%$ de los encuestados conocen de qué trata el secuestro como instrumento empleado por las redes organizadas para satisfacer la demanda de la sociedad de consumo, misma que es causa del delito de trata de personas en su modalidad de explotación sexual, el 10\% desconoce sobre el tema y al 2\% no le interesa conocer acerca de lo que trata.

En primer lugar, en concordancia con la figura en tratamiento podemos decir que el secuestro, como instrumento para satisfacer la creciente demanda de la sociedad de consumo, forma parte de la esfera de las conductas delictivas que la sociedad reprueba fehacientemente por cuanto implica la violación al derecho a la libertad, asimismo, mediante esta práctica las redes organizadas que constituyen la industria dedicada a la explotación sexual obtienen recursos que confrontan al Estado y a la sociedad atentado y transgrediendo los valores y principios de los mismos, ello de modo tal que, la inseguridad se visibiliza cada vez más y sensibiliza a la población en su conjunto respecto del acrecentamiento de la percepción de riesgo que dicha práctica implica (Gómez, 2004, pp.198-199).

La demanda por parte de la sociedad de consumo ha tenido un abrumador crecimiento en el siglo XXI puesto que la mercantilización de servicios sexuales y su vínculo con mercados de diversa índole (por ejemplo, turismo) han proporcionado las condiciones propicias y necesarias para la prestación de servicios sexoafectivos a una escala inimaginable (Agustín, 2000). En este negocio se cuenta con la participación directa de los ofertantes y demandantes, que configuran la sociedad de consumo, e indirecta de agentes sociales, actividades económicas legales, por ejemplo, el servicio de taxis, hoteles, e ilegales como el turismo sexual (Hurtado, 2018). Cabe precisar que la clientela de esta industria es increiblemente numerosa, de hecho se ha calculado que hasta, aproximadamente, un millón de hombres solicitan sexo todos los días en nuestro país, situación que resulta beneficiosa para las redes organizadas en materia de explotación sexual y desfavorable para la sociedad, ello en la medida que dicho crecimiento de la demanda implica el abastecimiento del mercado para lo cual los ofertantes harán uso de este y otros instrumentos para captar personas.

En segundo lugar, podemos decir que una gran cantidad de personas tiene conocimiento acerca del secuestro como medio para para satisfacer la demanda de la sociedad de consumo en la Región de Puno, lo cual resulta favorable para plantear y promover lineamientos preventivos que orienten el accionar de las autoridades competentes para el tratamiento del delito en tratamiento a fin de disminuir el grado de incidencia de la trata de personas en nuestra sociedad. 
De este modo, es menester mencionar que la sociedad de consumo ha tenido un creciente y progresivo desarrollo en nuestra Región, sobre todo en las minas, ello lo podemos contrastar directamente con las noticias de los distintos medios de comunicación, más no con los registros que ofrece el INEI, los Planes Nacionales referidos al tema y otros, puesto que en estos encontramos, lamentablemente, una abrumadora cifra negra que no nos permite conocer la realidad de la explotación sexual propia de la trata de personas y mucho menos de sus causas, lo cual entorpece y retrasa el trabajo multisectorial y descentralizado que vienen llevando a cabo el Gobierno Central, los Gobiernos Regionales y Locales, la Policía y otros actores imprescindibles para tal encomiable labor. Sobre la cifra negra en cuestión el INEI menciona que el año 2020 se han registrado 245 denuncias de la modalidad de explotación sexual referido a la trata, dicha cifra representa el $74 \%$ del total de denuncias del delito en cuestión.

$\mathrm{Al}$ respecto, cabe mencionar que, los llamados a combatir la presente problemática social, no son solo las autoridades, es decir, personas con algún cargo, sino también la sociedad en general, sin excepción alguna, puesto que sin su cooperación cualquier trabajo, por más bien elaborado que se encuentre, quedará inutilizado en el baúl de las buenas intenciones, por ello es importante que cada uno de nosotros tomemos parte y asumamos el compromiso de prevenir la explotación sexual, para ello y como primer paso, es importante pues que se cuente con la información adecuada en relación al tema, ya sea de lo que trata, de sus causas, de sus consecuencias y otros aspectos que pudiesen desprenderse del mismo, puesto que no hay mejor manera de hacer frente a esta problemática social que el de estar informados, no solo para realizar algún tipo de acción que contribuya con la causa, sino también para poder orientar a aquellos que desconocen sobre el tema.

\subsection{LAS POLÍTICAS PÚBLICAS PARA SU PREVENCION EN LA REGIÓN DE PUNO}

OBJETIVO N²: Analizar la aplicación de la política publicas contra la trata de personas en la región de Puno.

Las políticas públicas en general son documentos que se enmarcan en las políticas de Estado, estas son planteadas en base a la identificación de problemas o necesidades que tienen una sociedad, y deben ser priorizados por cada uno de los ministerios. En lo que respecta al problema de trata de personas se han formulado políticas públicas, estas en base a la búsqueda de disminución de casos sobre el tema referente. Lamentablemente en los últimos años este delito de trata de personas se ha intensificado considerablemente, según el INEI en el año 2018, hubo 734 denuncias y también el MININTER señalo que, de mayo del 2016 a mayo del 2020, prácticamente hay 4.877 víctimas relacionadas a la trata de personas, de los cuales el $71 \%$ de los casos se registra en las regiones de Lima, Madre de Dios, Puno y Tacna. Son todos estos hechos que nos hacen realizar un análisis sobre cómo es la aplicación de las políticas públicas en la región de Puno, para ello partiremos de dos premisas fundamentales, el conocimiento que tienen los principales agentes que hacen uso de este instrumento y también la apreciación practica que se tiene sobre las políticas públicas. 


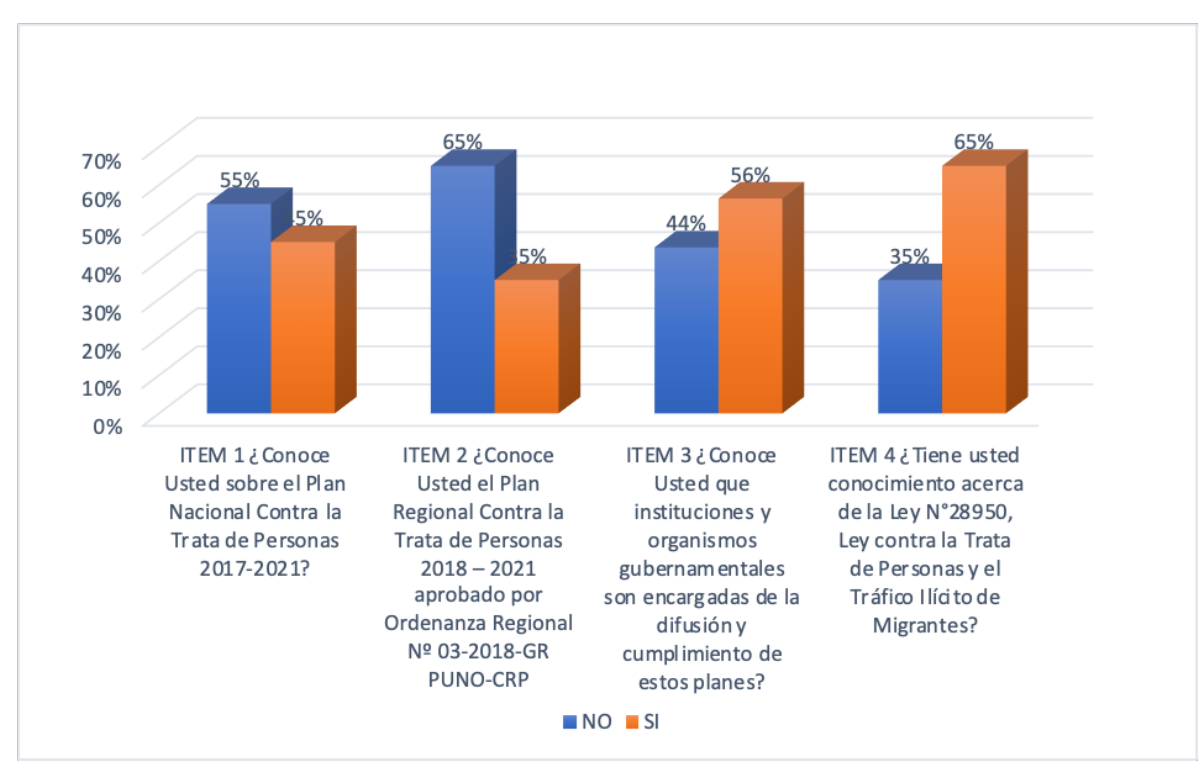

FIGURA 5

Conocimiento de las políticas públicas y leyes contra la trata de personas Nota. Encuesta elaborada por el equipo de investigación y realizada mediante Google Forms, Julio 2021.

\section{Interpretación}

Según la figura 5, de los 40 encuestados, se aprecia cuatro preguntas que tienen como objetivo principal, identificar el conocimiento que tienen la población materia de estudio, en este caso los trabajadores de los entes gubernamentales, los cuales se relacionan directamente con las políticas públicas, esto de acuerdo a las funciones que poseen las entidades. El primer ítem señala que $55 \%$ de la muestra materia de estudio no conoce el plan nacional contra la trata de personas 2017-2021, pero el $45 \%$ si conoce. El segundo ítem señala que $65 \%$ de la muestra materia de estudio no conoce el plan regional contra la trata de personas 2018-2021, mientras que el $35 \%$, si conoce. El tercer ítem señala que $44 \%$ de la muestra materia de estudio no conocen las instituciones y organismos gubernamentales que se encargan de la difusión y cumplimiento de los planes que se vinculan con las políticas públicas. El cuarto ítem señala que el $65 \%$ conoce la ley 28950 , mientras que el 35\% no conoce dicha ley que hace referencia a la trata de personas.

Con lo antes mencionado se puede deducir que las entidades gubernamentales encargadas de su creación, formulación, aplicación e implicación de acuerdo a la función de cada entidad, no conocen este implemento fundamental para la disminución de casos de trata de personas, es por tal motivo que señalamos como principal factor en contra el desconocimiento, ya que las políticas públicas en general tienen objetivo de solucionar un problema que aqueja a un sector vulnerable. 


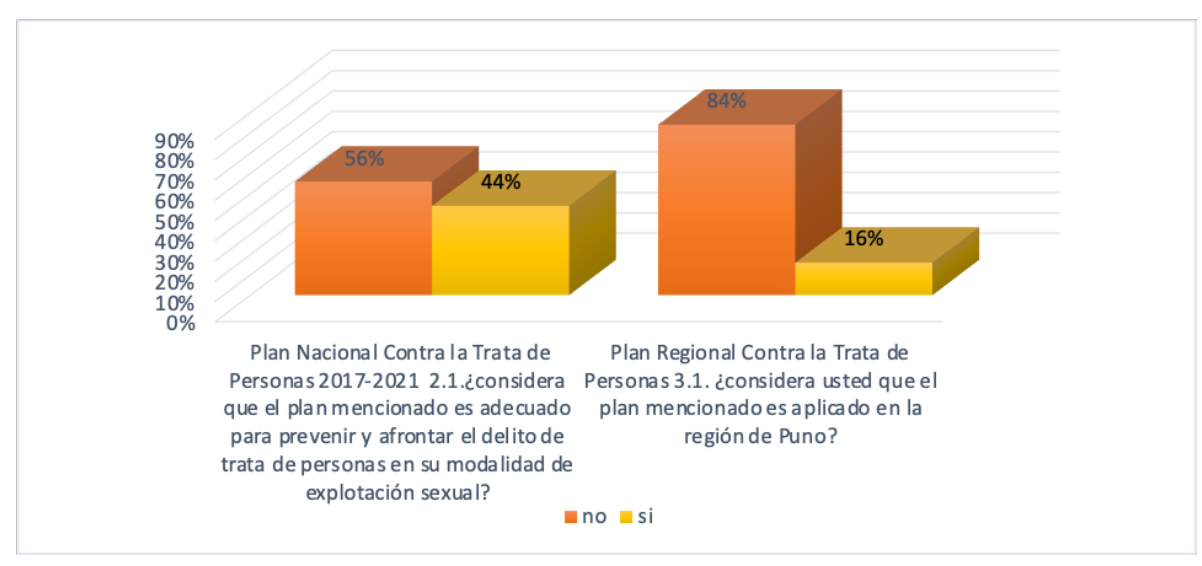

FIGURA 6

Aplicación del Plan nacional y Plan regional contra la trata de personas Nota. Encuesta elaborada por el equipo de investigación y realizada mediante Google Forms, Julio 2021.

\section{Interpretación}

Según la figura 6 , se puede observas que, de 25 encuestados que respondieron afirmativamente a la pregunta sobre si tienen conocimiento de la existencia de los planes, dan como resultado en el primer ítem 56\% considera que el plan nacional contra la trata de personas es adecuado para prevenir y afrontar el delito de trata de personas, mientras que el $44 \%$ señala que el plan no es adecuado. El segundo ítem señala que $84 \%$ de encuestados considera que el plan regional contra la trata de personas no es aplicado en la región de Puno y solo el 16\% de encuestados considera que si es aplicado. Uno de los principales problemas de los entes gubernamentales es la función que tienen con las políticas públicas que se crean, ambos planes tanto nacional y regional tienen carencias, no solo aplicables, sino de estructura y desarrollo, es por tal motivo que se busca analizar los planes en miras de plantear lineamientos de políticas públicas preventivas para anticipar la consumación del delito.

Posibles causas de una aplicación ineficiente:

\subsubsection{Enfoque descentralizado}

Se evidencia la falta de compromiso de los gobiernos regionales al no responder las solicitudes de información o enviar respuestas donde se afirma que en sus jurisdicciones no se han registrado casos de trata, olvidando que existen acciones de prevención que podría desarrollarse. Incluso en las regiones donde existen Planes Regionales de Lucha contra la Trata de personas, siendo así que el avance de su implementación no ha sido informado como se debe. Esto en General, es preocupante el bajo nivel de involucramiento de los gobiernos regionales y municipales en las acciones contra este delito, teniendo en cuenta que son las instancias en representación del Estado que por su ubicación territorial estarían de alguna manera en mejores condiciones de poder identificar a las poblaciones y demás situaciones de riesgo, así como, prevenir y brindar una atención oportuna a las víctimas y poder coordinar con las demás instancias del Estado la reintegración de las sobrevivientes de este delito.

\subsubsection{Carencia de un plan estructurado de monitoreo y seguimiento}

Si bien conocemos que el Plan Nacional contra la Trata de Personas 2017-2021 no ha logrado obtener el realce y funcionamiento como se esperaba en un inicio, este plan que busca la prevención no ha sido uno de los planes que haya llegado a la meta con la cual en un inicio se pretendía, la cual era la prevención y afronte 
de este delito que es la trata de personas en su modalidad de explotación sexual. Esto debido a que existe un cierto tropiezo a la hora de querer hacer funcionar un plan, tropiezo que es a causa de la carencia de un correcto monitoreo y seguimiento estructurado del plan que se quiere hacer funcionar.

Como bien se menciona que la evaluación del progreso del plan, el monitoreo de las incidencias que presenta dicha estrategia lleva consigo que se pueda visualizar los cambios que está generando esta en el panorama general al cual se pretende influir; todo esto debido a que si se tiene una continua evaluación y un buen monitoreo, permitirá identificar los riesgos o fallas que se está presentando para con ello poder afrontarlos antes de que se conviertan en obstáculos del resultado que se pretende obtener. (SOMO, 2021)

Es por todo ello que, si un plan no tiene avances, una de las causad puede llegar a ser no se presta monitoreo de este plan, no se presenta la real importancia que se merece y aún se presenten fallas en el monitoreo y seguimiento, en lo más pronto podrán solucionarse y obtener la eficacia con el cual se contó que se obtendría en un inicio.

Por ello, como expresión de todos los sectores del estado y de la sociedad involucrada, es cierto que no pude realizarse una evaluación periódica conjunta de los avances del plan, sin embargo, se puede lograr un monitoreo por departamento, mayormente los distritos más golpeados por los altos casos de Trata de Personas en su modalidad de explotación sexual, con el fin de demostrar si se están presentando fallas y/o avances de eficacia del mencionado plan.

\subsubsection{Debilidad de los compromisos presupuestales}

El ministerio del interior ratifica su lucha contra la trata de personas en el Perú asignando para el 2018, un presupuesto aproximado de $s / 7,5$ millones, según el vi informe de chs alternativo, similar inversión es destinado para combatir este delito en el año 2019 que es aproximadamente $\mathrm{S} / 7^{\prime} \quad 758.410,81$ establecido en el vii informe alternativo, realizado por esta ONG. Y actualmente contamos con un plan nacional contra la trata de personas 2017 -2021 que se ve que aún falta financiar el presupuesto para poder ejecutar el plan nacional de lucha contra este delito pues según las estadísticas se ve que nadie conoce este plan además de ello nuestras autoridades como la policía nacional del Perú no se les brinda alguna capacitación relacionada con este plan entonces podemos decir que obviamente un plan regional no va tener la solvencia necesaria para tratar de combatir este delito por ello es necesario destinar más presupuesto que es lo que hace falta para poder desarrollar las habilidades de los operadores de la justicia y así llevar este tema no solo a nivel nacional sino extendernos a las regiones para hacer mesas de coordinaciones con cada zona y de conformidad a la realidad en que se encuentran aplicar los planes que se tienen para combatir ese delito; capacitar sobre el tema a las autoridades, crear albergues para las víctimas que han sufrido la trata y son rechazadas por la sociedad y por sus familiares y así impedir que vuelvan a esos lugares pues con ese poco presupuesto que se brinda no es posible solventar todo ello por eso sería fantástico que el estado a través de la MEF pueda poner en marcha un programa presupuestal para así combatir este delito y solventar este plan nacional en su totalidad.

\subsubsection{DISPOSICIONES IMPOSIBLES DE CUMPLIR}

El Plan Nacional contra la Trata de Personas 2017-2021 por medio de sus objetivos y metas ha proyectado la lucha contra esta problemática social de manera ideal puesto que los preceptos aducidos y establecidos en dicho texto resultan ser incongruentes con el plano de la realidad que requiere de un abordaje distinto al planteado. La necesidad de un tratamiento adecuado que permita disminuir los casos de trata de personas es notable, sin embargo, ante tal situación, nuestras autoridades no tienen mejor idea que teorizar dicha disyuntiva y dejar que estos planes sean olvidados en el tiempo. 
El cumplimiento del actual Plan se hace sumamente difícil de lograr debido a la insuficiencia y falta de acuerdos bilaterales y multisectoriales, integralidad y enfoque sistémico, estrategias integrales de persecución, fiscalización y sanción, servicios de protección y reintegración, débil, de gestión del conocimiento, entre otros.

\section{PLANTEAMIENTO DE LOS LINEAMIENTOS PARA LA POLÍTICA PUBLICA}

Para Thomas Dye (1987), quien plantea que las Políticas Públicas son “todo lo que los gobiernos deciden hacer o dejar de hacer”. Entonces decimos que, son aquellas propuestas de solución a problemas de carácter meramente públicos, ya que, los problemas de una colectividad se convierten en públicos cuando la solución de estos mismos correspondería legalmente a un órgano del gobierno. Entonces entendamos a una política pública como aquel conjunto de objetivos, decisiones y acciones que van a ser realizadas por un Gobierno, con el fin de dar solución a dichos problemas que aqueja a la ciudadanía.

Para Von Liszt citado en Muñoz (2011) la política criminal es un conjunto sistemático de principios basados en la actividad científica- investigativa tanto de las causas de los delitos e infracciones como de eficacia de la pena, mediante los cuales el Estado direcciona la lucha contra la criminalidad a través de la pena y sus derivados (pp.130).

Decidimos escoger la Política pública, puesto que, el delito de trata de personas con fines de explotación sexual, es un problema social de suma importancia tanto para la ciudadanía como para el estado, ya que, la política pública implica la elaboración de acciones orientadas a metas y objetivos particulares, como lo es en este caso.

\subsection{Lineamientos para una política pública preventiva}

La trata de personas en su modalidad de explotación sexual es un problema que acarrea a todo el país; los departamentos, gobiernos regionales e incluso los diversos entes que hacen posible que diversas políticas públicas que prevén este delito se pongan en funcionamiento. Por ello es que se planteó una encuesta para obtener una respuesta de qué es lo que posiblemente se necesita para afrontar este delito y es por la justificación mencionada que se plantearon lineamientos preventivos para afrontar el delito de trata de personas en su modalidad de explotación sexual, lineamientos que se basan en prevenir que las víctimas puedan ser captadas, tomar medidas psicológicas que ayuden a prevenir la incurrencia inocente de las víctimas al momento de que creer en los victimarios.

La encuesta se basó en que los participantes respondieran la cuestión sobre, cuál sería la mejor manera de afrontar el delito de trata de personas en su modalidad de explotación sexual, se contó con la participación de 270 participantes que demostraron que una de las mejores maneras de afrontar este delito es el mayor control y fiscalización además de promover una educación preventiva, prevención que según los participantes ayudaría a afrontar este delito; de manera psicológica y también prevenciones que reforzarían la grave afectante que es la economía.

Según un estudio, nos indica que la prevención puede ser efectiva y puede generar el grado alto de cumplimiento de parte de la ciudadanía frente a las leyes, debido a su baja inversión económica o como se menciona, los costos de inversión son mínimos y son medidas que pueden llegar a lograr un grado alto de efectividad. (Shaw, 2011)

LINEAMIENTO $\mathrm{N}^{\circ} 1$

A. Promover e implementar la secundaria técnica para facilitar el acceso de jóvenes al mundo laboral

- los jóvenes si tuvieran una formación técnica que les permita poder generar ingresos, ya no buscarían trabajos con desesperación y ser presa fácil para la captación de trabajos de procedencia oscura

- La meta es que todos los peruanos accedan a servicios de educación técnica productiva

- Con ayuda de (MINEDU) se puede impulsar que se brinde una secundaria técnica para así los jóvenes al momento de culminar la secundaria tengan herramientas para que les permita abrirse puertas en el mundo laboral 
- Para que esto sea posible es necesario disponer de un financiamiento, para la implementación de la infraestructura y equipamiento de formación técnica en todos los colegios, con el fin de desarrollar capacidades tecnológicas productivas de conformidad con las particularidades de cada región

- Por ello sería fantástico que el estado a través de la MEF en coordinación con la MINEDU pueda poner en marcha un programa presupuestal para así implementar la secundaria técnica para facilitar acceso de jóvenes al mundo laboral y reducir la desaparición de jóvenes por haber sido captados por falsas propuestas de trabajo y ser víctimas de la trata de personas en su modalidad de explotación sexual y obviamente tan bien se va a reducir la pobreza.

B. Creación de agencias de trabajo conjuntamente con La Dirección Regional de Trabajo y Promoción del Empleo Puno bajo la supervisión del SUNAFIL

La finalidad de la creación de agencias de trabajo es poner en marcha un servicio dirigido a favorecer, por una parte, la creación de empleo y por otra, mejorar la formación y orientación. Otra de las finalidades de la creación de una agencia de trabajo es dar la seguridad tanto a empleadores como a los futuros empleados que solicitan alguna propuesta laboral, así como también ampliar las oportunidades de acceder a un empleo decente, mejorar la protección social y fortalecer el diálogo al abordar temas relacionados con el trabajo. Sobre los objetivos, planificar y desarrollar las políticas de acción que permitan impulsar las iniciativas laborales de manera segura, decimos de una manera segura, puesto que, la seguridad es primordial al momento de conseguir trabajo, ya que muchas veces las propuestas laborales que se brindan pueden derivar en delitos tal como la trata de personas. La disminución de la tasa de desempleo y por último el aumento de la cualificación tanto de los desempleados como de los empleadores.

\section{LINEAMIENTO N²}

A. Extender las redes de la capacitaciones y charlas sobre lo que implica la trata de personas en la modalidad de explotación sexual.

Lo que se busca con este lineamiento es la propuesta de extender las redes de capacitación a los lugares más recónditos del país; capacitación que será brindada por psicólogos y por efectivos de la Policía Nacional del Perú es por ello que se contará con el apoyo del Ministerio Publico quien, con la mano del Ministerio de Salud, brindarán personal de salud como psicólogos capacitados anteriormente sobre el tema de trata de personas en su modalidad de explotación sexual y en el tema de la participación de efectivos policiales es que se buscará el apoyo del Ministerio del Interior, para que brinde capacitación anterior a dichos efectivos con el fin de que después de ello, estos estén capacitados para brindar dichas charlas a la población. Efectivos de la Policía Nacional del Perú también participarán en dicha capacitación, efectivos que junto a un miembro policial especializado en casos denunciados por trata de personas en su modalidad de explotación sexual brinden esta información a la ciudadanía, información que puede constar acerca de las modalidades de captación por la cual pasaron las victimas que denunciaron tal acto delictivo como es la trata de personas en su modalidad de explotación sexual.

Esto con el objetivo de prevenir psicológicamente a la población para que conozcan la fácil captación de personas por redes organizadas, esto mostrando a las personas como actúan dichas redes, cuáles son sus modalidades o principalmente, como hacen estos captores para llegar a sus víctimas. Como bien dimos a entender en apartados anteriores que, una de las causas para que ocurra la trata de personas en su modalidad de explotación sexual era la existencia de una sociedad de consumo y que estas por medio de redes organizadas, captaban víctimas para proveerlas a dichos consumidores, victimas que pueden ser captadas por su inocencia e ignorancia acerca de este tema, entonces para prevenir dicha captación es que se brindará la información psicológica a la población; información que derivara en cómo una persona debe de actuar si presencia un acto de amenaza, seducción e incluso un comportamiento que imparta pensar en un futuro secuestro. Es entonces que las personas psicológicamente se les dará charlas de qué es lo que pasa en la trata de personas en su modalidad de explotación sexual, se les brindará apoyo emocional al igual que sentimental. Lo siguiente es que los efectivos policiales, juntamente con un efectivo encargado en recibir denuncias acerca de la trata 
de personas en su modalidad de explotación sexual, brinden detalles de cómo es la captación de las personas frente a este tema, también que impartan conocimiento de la gravedad de este delito al igual que nos brinden información de cuantos son los casos denunciados en nuestra región y en nuestro país. El apoyo de ambos capacitadores, será de gran ayuda para prevenir este delito y que lo principal es buscar que las personas no sean inocentes e ignorantes con este tema que es de gran trascendencia como la trata de personas en su modalidad de explotación sexual.

\section{CONCLUSIONES}

Respecto al primer objetivo que nos hemos planteado en un principio y desarrollado a lo largo de la investigación, podemos concluir que la causa principal es el factor económico, el cual, fue confirmado por medio de la encuesta realizada a la población de la ciudad de puno, obteniendo un resultado desfavorable, puesto que, la gran mayoría podría ser víctima potencial del delito de trata de personas en su modalidad de explotación sexual.

Con respecto del segundo objetivo trazado en el presente trabajo de investigación, también llevada a cabo mediante una encuesta sobre el conocimiento de los planes y/o políticas públicas referidas a la prevención del delito de trata de personas en su modalidad de explotación sexual, dirigidas a los principales órganos gubernamentales en quienes recaen el correcto funcionamiento de estos planes y/o políticas públicas, arrojando un resultado poco favorable, puesto que, una gran mayoría desconocía acerca de estos planes y la minoría que si tenía conocimiento sobre dichos planes, deducían que estos planes no funcionaban debido a que fallas e irregularidades a la hora de poner en marcha dichos planes.

Es por las dos conclusiones mencionadas en líneas anteriores, que producto de la investigación presente, es que nosotros a partir de ellos, planteamos lineamientos los cuales están dirigidos a la prevención del delito de trata de personas en su modalidad de explotación sexual. Lineamientos que puestos en un correcto funcionamiento y monitoreo del mismo lograran que las causas primordiales objeto de la presente investigación tengan como resultado una notable reducción.

\section{REFERENCIAS BIBLIOGRÁFICAS}

Alfaro Calvo, F. (2003). Explotación sexual comercial. Costa Rica: OIT. http://white.lim.ilo.org/ipec/documentos/p a_documentos_de_trabajo.pdf

Bernal Camargo, D., Varón Mejía, A., Becerra Barbosa, A., Chaib-De Mares, E., \& Archilla Delgado, L. (2013). Explotación sexual de niños, niñas y adolescentes: modelo de intervención. Latinoamericana umanizales, XI(2), 617-632. http://www.scielo.org.co/pdf/rlcs/v11n2/v11n2a12.pdf

Carrasco Yerro, E. (Marzo de 2017). Guia definitiva para encontrar empleo: Infoempleo. https://blog.infoempleo.com /media/2017/03/guia-encontrar-trabajo-2017-infoempleo.pdf

Chs- alternativo . (2010). La trata de personas en el Perú . 1-47.

Comisión Europea. (2010).La Trata de Personas en el Perú Manual Para Conocer el Problema. Lima.

De Gonzáles Mariscal, O. (2002). El Secuestro: Análisis Jurídico. Biblioteca Jurídica Virtual del Instituto de Investigaciones Juridicas de la UNAM, 53-134. https://archivos.juridicas.unam.mx/www/bjv/libros/1/244/3.p df

Hurtado Saa, T. (2018). La producción social del mercado del sexo y de la ocupación de trabajadoras sexuales en España. Revista Colombiana de Antropología. (35-58), http://www.scielo.org.co/pdf/rcan/v54n2/0486-6525-rcan-54 -02-00035.pdf

Gómez Sánchez, L. (2004). Sociología de la violencia. El secuestro, empresa parapolicial, vol.10, http://www.scielo.org $. \mathrm{mx} /$ scielo.php?script $=$ sci_arttext\&pid=S1405-74252004000200011

Gobierno Regional de Puno. (2018). Plan Regional contra la trata de personas 2018-2021. Puno. 
Gobierno Regional de Loreto. (2013). Plan regional de acción contra la trata de personas loreto 2013-2017.

Ley $\mathrm{N}^{\circ} 28950$ del 2016. Ley contra la trata de personas y tráfico ilícito de migrantes. D.S. N001-2016-IN

Lijtenstein Morganti, M. (2018). Violencia y abuso sexual en la niñez y la adolescencia. Sugia, VII(3), 1-94. http://ww w.codajic.org/sites/www.codajic.org/files/Revista\%20Sugia\%202018\%20Vol\%207-3.pdf

Muñoz Tejada, J. (2011). La politica criminal: creencias, discursos, prácticas saber y poder. Revista Nuevo Foro Penal, 130,

Organización de las Naciones Unidas. Protocolo para prevenir, reprimir y sancionar la trata de personas, especialmente mujeres y niños. (1 de febrero del 2000)

Organización de las Naciones Unidas. Declaración de Nueva York sobre refugiados y migrantes . Asamblea General. ( 17 de junio del 2016)

Querol Lipcovich, A. (2007). La Trata de Personas en el Perú. Lima: Just Print SAC. https://www.trabajo.gob.pe/m igrante/pdf/trata_personas_peru.pdf

The Naked Anthropologist. ( 15 de Agosto de 2008). Trabajar en la Industria del sexo, https://www.lauraagustin.co $\mathrm{m} /$ trabajar-en-la-industria-del-sexo

Shaw, M. (2011). Manual sobre la aplicación eficaz de las Directrices para la prevención del delito. New York: Naciones Unidas. https://www.unodc.org/documents/justice-and-prison reform/crimeprevention/ Handbook_on_the_Crime_Prevention_Guidelines_Spanish.pdf

Somo.(6 de Agosto de 2021). Centre for Research on Multinational Corporations. https://webcache.googleusercontent. com/search?q=cache:SfG5NLPjO0YJ:https://taxjusticetoolkit.org/es/como-desarrollar-la-estrategia-de-incid encia-politica-en-tributacion/paso-6-planificar-el-monitoreo-y-evaluacion $/+\& c d=1 \&$ hl=es-419\&ct $=$ clnk\&gl $=$ pe

\section{INFORMACIÓN ADICIONAL}

Investigación formativa: Investigación realizada por estudiantes del curso de Criminología de la Escuela Profesional de Derecho de la Universidad Nacional del Altiplano de Puno. 